\title{
INFLUÊNCIA DAS TÉCNICAS DE TERAPIA MANUAL OSTEOPÁTICA NA FUNÇÃO RESPIRATÓRIA
}

\author{
Jefferson Jovelino Amaral dos Santos \\ Marcelo Claudio Amaral Santos ${ }^{2}$ \\ Junior De Carli \\ Priscila Rocha ${ }^{4}$ \\ Keyla Elverita Klein Previatti ${ }^{5}$
}

SANTOS, J. J. A. dos; SANTOS, M. C. A.; CARLI, J. de; ROCHA, P.; PREVIATTI, K. E. K. Influência das técnicas de terapia manual osteopática na função respiratória. Arq. Cienc. Saúde UNIPAR, Umuarama, v. 19, n. 3, p, 191-197, set./dez. 2015.

\begin{abstract}
RESUMO: As técnicas de terapia manual são consideradas como um método sistemático de avaliação e tratamento das disfunções do sistema neuromusculoesquelético, e estas têm como finalidade principal recuperar o movimento fisiológico em áreas onde existe restrição ou disfunção melhorando a função dos sistemas adjacentes. Contudo, na literatura são escassos os estudos sobre a utilização e eficácia destas técnicas, seja manipulativa ou de mobilização, na função respiratória, principalmente como uma forma preventiva de tratamento. O objetivo deste estudo foi avaliar as alterações nas funções pulmonares e na expansibilidade torácica em indivíduos normais submetidos às técnicas osteopáticas. Este estudo foi caracterizado como quase experimental. Fizeram parte da amostra 30 voluntárias, com idade de 18 à 28 anos, sem patologias pulmonares conhecidas associadas. A avaliação da função pulmonar e expansibilidade torácica foram por meio da espirometria e cirtometria respectivamente. A amostra recebeu procedimento de intervenção sendo avaliada em dois momentos, antes e após a aplicação de uma manobra osteopática de manipulação (thrust) em coluna torácica e técnica de músculo energia para peitorais. Os resultados indicam que o protocolo foi capaz de aumentar a mobilidade torácica em todos os parâmetros avaliados, e na função pulmonar houve melhora estatisticamente significativa no pico de fluxo expiratório.
\end{abstract}

PALAVRAS-CHAVE: Terapia Manual; Manipulação Torácica; Espirometria; Cirtometria Torácia.

\section{INFLUENCE OF OSTEOPATHIC MANUAL THERAPY TECHNIQUES IN THE RESPIRATORY FUNCTION}

\begin{abstract}
Manual therapy techniques are considered to be a systematic method of assessment and treatment of disorders of the neuromusculoskeletal system, and are intended to recover the main physiological movement in areas where there is restriction or dysfunction, by improving the function of adjacent systems. However, in literature, there are few studies on the use and effectiveness of such techniques, whether by manipulative or mobilization techniques, in respiratory function, especially as preventive treatment. The aim of this study was to evaluate changes in lung function and chest expansion in normal individuals subjected to osteopathic techniques. This study was characterized as quasi-experimental. The sample consisted of 30 volunteers, aged 18 to 28 years, without known lung diseases associated. The evaluation of lung function and chest expansion were calculated using spirometry and circumference measurements, respectively. The sample received intervention procedure and was evaluated in two phases, before and after applying a move osteopathic manipulation (thrust) in the thoracic spine and the muscle energy technique for pectoral muscles. The results indicate that the protocol was able to increase thoracic mobility in all parameters, and lung function was statistically significantly improvement in peak expiratory flow.
\end{abstract}

KEYWORDS: Manual Therapy; Thoracic Manipulation; Spirometry; Thoracic Expansion.

\section{Introdução}

Para Leite (2000), o movimento humano depende da transformação da energia química dos nutrientes em energia mecânica, de maneira especial, por meio do metabolismo aeróbico. A partir disso, o fornecimento de $\mathrm{O} 2$ e a remoção do $\mathrm{CO} 2$ acontecem durante a respiração pelo enchimento e esvaziamento dos pulmões por dois mecanismos: a) pelos movimentos do diafragma para cima e para baixo, que fazem com que a caixa torácica se encurte ou se alongue (GUYTON; HALL, 2002); b) pelo alargamento toráciconos movimentos de alça de balde e braço de bomba (KAPANDJI, 2000).

Para a realização desses movimentos os músculos respiratórios precisam vencer a resistência do pulmonar, do gradil costal e das vias aéreas no sentido de expandir a caixa torácica e criar uma pressão negativa intra-pulmonar (KENDALL; McCREARY; PROVANCE, 1995).

Em decorrência da musculatura respiratória estar sempre ativa, e por participar da manutenção do tórax, esta apresenta características de musculatura tônica e, com frequência, está retraída, limitando consideravelmente as possibilidades inspiratórias, sugerindo menor oferta de oxigênio celular (SOUCHARD, 1996). Yamaguti et al. (2007) também mostraram que existem diferenças na mobilidade diafragmática conforme o posicionamento mesmo em indivíduos saudáveis.

Farkas, Cerny e Rochester (1996) ressaltam que além de compartilhar todas as características comuns aos músculos do esqueleto apendicular, os músculos respiratórios estão propensos a se fadigarem e também estão dotados com a capacidade de se adaptarem a condições adversas, incluindo os exercícios físicos, que podem levar ao encurtamento dos músculos inspiratórios acessórios.

Souchard (1996) menciona que os músculos inspiratórios, ao se encurtarem, aumentam o diâmetro da caixa torácica em repouso. Um tórax bastante desenvolvido aparenta grande capacidade respiratória, porém Guyton e Hall

DOI: https://doi.org/10.25110/arqsaude.v19i3.2015.2873

${ }^{1}$ Professor Titular do Curso de Fisioterapia Unipar. Osteopata - IDOT. Fisioterapeuta Reab Plus Fisioterapia Toledo-PR. Brasil. R. Barao do Rio Branco 1594 - Toledo - PR. email: jeffe@unipar.br

${ }^{2}$ Professor do Curso de Fisioterapia UNOESTE. Presidente Prudente-SP. Brasil

${ }^{3}$ Osteopata - IDOT. Brasil.

${ }^{4}$ Acadêmica do Curso de Fisioterapia - UNIPAR. Brasil

${ }^{5}$ Acadêmica do Curso de Fisioterapia - UNIPAR. Brasil 
(2002), afirmam que um aumento do diâmetro da caixa torácica aumenta o volume residual e diminui o volume corrente, minimizando assim a troca gasosa.

Bienfait (1995) relata que ao se tornar mais curto o tecido perde sua elasticidade e deixa de preencher com perfeição sua função mecânica. Quanto mais elasticidade o tecido perde, mais tensionamento ele suporta, e consequentemente se densifica, criando, assim, um ciclo vicioso. Souchard (1996) corroborando, acredita que estirar a musculatura estática seja uma necessidade. Mais recentemente, Clanton e Levine (2009) mostraram que o encurtamento do diafragma é evidente em casos de doença pulmonar obstrutiva crônica em decorrência a hiperinsuflação prolongada e que o estiramento das fibras pode melhorar a efetividade das contrações.

A base teórica para a ação da osteopatia tratamento manipulativo e seu efeito no corpo foi avançada com base na ativação autonômica, causando vasodilatação concomitante do músculo liso relaxamento e aumento do fluxo sanguíneo, resultando em melhor amplitude articular, diminuição da percepção da dor, e / ou alteração no tecido (HENLEY et al., 2008; LOMBARDINI et al., 2009).

Na maioria das vezes a técnica osteopática de thrust envolve uma alta velocidade de impulso, uma técnica em que as articulações são ajustados rapidamente, muitas vezes acompanhada de estalidos. Os osteopatas acreditam que, muitas vezes, um estalido acústico associado a uma manipulação de impulso é um critério para determinar o sucesso da técnica (CLELAND et al., 2007).

Durante a manobra, a pressão é exercida sobre a biomecânica, quer por meio de um longo braço de alavanca, em que força é aplicada distante do comum, ou um braço curto da alavanca, quando a força é aplicada junto ao conjunto (ERNST, 2007).

Para a constatação desses fatos, faz-se necessário a utilização de uma forma de mensuração que apure a eficácia dessas técnicas na performance respiratória, para este fim empregou-se as provas espirométricas, e medidas das circunferências toracoabdominais.

Pereira (2002) descreve que por meio da espirometria, é possível medir o volume de ar inspirado e expirado e os fluxos respiratórios, sendo especialmente útil a análise dos dados derivados da manobra expiratória forçada. A espirometria $($ do latim spirare $=$ respirar + metrum $=$ medida $)$ é um exame peculiar que exige a compreensão e colaboração do paciente, equipamentos exatos e emprego de técnicas padronizadas aplicadas por pessoal treinado, em que os resultados obtidos devem ser comparados à valores previstos adequados para a população avaliada de acordo com o mesmo autor.

A interpretação da espirometria é baseada em bom conhecimento da arquitetura pulmonar, ontogenia e fisiologia respiratória (ANOGEIANAKI; NEGREV; ILONIDIS, 2007). Ochs et al. (2004) demonstraram que o número médio alveolar é de 274 a 790 milhões e que o número total alveolar está intimamente relacionado ao volume pulmonar total, estima-se que a área total de superfície alveolar é de 70 a 120 metros quadrados, ou cerca de 70 vezes maior que a área de superfície corporal e que a superfície alveolar correlaciona-se positivamente com a taxa metabólica, independentemente do tamanho do corpo, enquanto que o volume pulmonar correlaciona-se positivamente com a altura do indivíduo.

Com este exame, basicamente são medidos alguns dos volumes pulmonares, particularmente a capacidade vital, e os fluxos aéreos, sendo os mais destacados segundo Silva (2005), a capacidade vital forçada (CVF), que corresponde ao volume expirado com esforço máximo e o mais rapidamente possível, a partir de uma inspiração máxima; volume expiratório forçado no primeiro segundo (VEF1), que corresponde ao volume expirado no primeiro segundo da CVF; pico de fluxo expiratório (PFE), que indica o fluxo máximo obtido logo após o início da CVF (geralmente ao final de 0,2 segundo e depende da colaboração do paciente).

A cirtometria ou perimetria toracoabdominal consiste em um conjunto de medidas das circunferências de tórax e abdômen durante os movimentos respiratórios, sua finalidade é avaliar a expansibilidade torácica de forma simples e acessível, esta técnica vem sendo referenciada como parâmetro de mensuração da expansibilidade pulmonar, além de ser utilizada com o objetivo de avaliar outros parâmetros amplitude torácica, volumes e capacidades pulmonares, complacência pulmonar, mecânica toracoabdobinal, função diafragmática, trabalho muscular e dispneia (CALDEIRA et al., 2007).

Segundo Costa et al. (2009), a cirtometria é considerada uma medida válida para a exploração das dimensões e amplitudes dos movimentos torácicos e abdominais, e tem sido muito utilizada para avaliar a mobilidade do gradio costal e do abdome durante os movimentos respiratórios.

Há muitos estudos que relatam a eficácia das técnicas de terapia manual na cervicalgia, lombalgia, nas mudanças das propriedades viscoelásticas dos tecidos, porém, na literatura são escassos os estudos sobre a utilização e eficácia das técnicas da terapia manual, seja manipulativa ou de mobilização, na musculatura respiratória, principalmente como uma forma preventiva de tratamento. Desta forma, justifica-se o interesse em pesquisar técnicas fisioterapêuticas manuais para a aplicação preventiva e, se estas influenciam a mecânica respiratória a ponto de potencializar a performance respiratória.

Portanto, o propósito deste estudo foi avaliar a função pulmonar em indivíduos normais submetidos às técnicas osteopáticas.

\section{Materiais e Métodos}

\section{Caracterização do estudo}

Esta pesquisa foi aprovada pelo Comitê de Ética em Pesquisas Envolvendo Seres Humanos (CEPEH) da Universidade Paranaense (UNIPAR) sob o protocolo 15370/2009 e caracterizou-se como um estudo quase experimental (THOMAS E NELSON, 2002).

\section{Amostra}

Fizeram parte da amostra 30 voluntárias de idade entre 18 a 28 anos, e que não apresentavam patologias pulmonares conhecidas com biotipo normolíneo.

\section{Critérios de inclusão e exclusão}

Os critérios de inclusão foram interesse e disponibilidade para participar da pesquisa, ter idade entre 18 e 28 
anos, voluntárias do gênero feminino, não apresentar patologias pulmonares conhecidas em seu histórico, indivíduos lúcidos e colaborativos para a compreensão das manobras a serem realizadas.

Foram excluídos do trabalho indivíduos menores de 18 anos e maiores de 28 anos de idade, indivíduos do gênero masculino, pessoas com histórico de patologias pulmonares, tabagistas, gestantes e aqueles que não conseguiam realizar as provas pulmonares solicitadas, assim como também os que se negaram a participar do estudo.

\section{Materiais}

Foram utilizados um monitor de pressão arterial digital de pulso (Z-40,Techline, Brasil) uma balança digital portátil (Plenna, Brasil) com capacidade de até $150 \mathrm{~kg}$, uma fita métrica convencional de 1,50 m, um espirometro digital (Pony Spirometer Graphic, Cosmed Srl, Itália).

\section{Procedimentos Metodológicos}

Todos os integrantes foram esclarecidos sobre os objetivos e os procedimentos realizados durante o desenvolvimento do estudo. Aqueles que aceitaram participar assinaram o termo de consentimento livre e esclarecido, a seguir foram encaminhadas para a realização de uma triagem para verificar se os indivíduos estavam hábeis e dentro dos parâmetros estabelecidos pela pesquisa.

Após a confirmação dos pré-requisitos que incluíam as voluntárias a participação da pesquisa, essas foram orientadas a permanecerem por um período de cinco minutos sentadas em repouso para depois iniciarem os testes.

No início do estudo os indivíduos tiveram suas medidas antropométricas registradas. Os indivíduos foram pesados com roupas leves, sua estatura foi obtida por meio da mensuração com o indivíduo sem sapatos, e a idade considerada foi a do seu último aniversário. As provas espirométricas foram realizadas após o indivíduo repousar durante 5 minutos, em ambiente calmo, e privado. Os dados coletados foram registrados em uma ficha especialmente desenvolvida para este estudo.

A amostra recebeu procedimento de intervenção, sendo que cada indivíduo foi avaliado em dois momentos: em primeiro instante foi avaliada a função pulmonar por meio da espirometria, e a expansibilidade toracoabdominal por meio da cirtometria, sendo que o indivíduo não havia recebido nem um tipo de interferência. Após a realização das provas pulmonares, e da cirtometria, os indivíduos eram orientados a permanecerem sentados e em repouso por cinco minutos, após o período, os indivíduos recebiam uma manobra osteopática de manipulação em coluna torácica ao nível de T6, e alongamento de músculos peitorais pela técnica de músculo energia e realizavam novamente as provas espirométricas por uma das quatro avaliadoras previamente treinadas, sendo que o período de intervenção de uma única sessão com duração 45 minutos.

Os indivíduos foram submetidos à avaliação espirométrica e de cirtometria toracoabdominal, na Clínica Escola de Fisioterapia da Universidade Paranaense - UNIPAR, Campus Toledo.

Todas as voluntárias fizeram uso do clipe nasal, fo- ram orientadas a permanecer em posição bípede, a realizar uma inspiração profunda para atingir a capacidade pulmonar total, que não deveria ser muito rápida, e a pausa pós-inspiratória não deveria exceder 3 segundos, pois isso causaria broncodilatação, alteração na retração elástica do pulmões e da parede tóracica. $\mathrm{O}$ tubete deveria ser mantido próximo à boca e nela colocado imediatamente após o término da inspiração máxima, sobre a língua, entre os dentes e lábios cerrados, evitando-se vazamentos, e a mão do indivíduo não poderia obstruir a válvula na parte posterior da turbina. Sendo que o indivíduo foi estimulado verbalmente e continuamente durante as provas pelas avaliadoras.

A duração da expiração forçada foi orientada a ser de no mínimo 6 segundos, sendo que para evitar erro de aprendizagem quando o último valor obtido for maior ao seu antecedente, a prova espirométrica era repetida. A aceitabilidade para o final do teste compreendeu a interrupção pelo próprio paciente devido a desconforto.

A expansibilidade toracoabdominal foi avaliada por meio da cirtometria. As voluntárias foram posicionadas em posição bípede e utilizando-se uma fita métrica convencional de $1,50 \mathrm{~m}$ foram tomadas as medidas das circunferências das regiões axilar (logo abaixo das axilas), basal (no final do apêndice xifoide) e abdominal (linlha umbilical). Cada medida foi obtida após solicitar ao paciente que realizasse uma inspiração máxima seguida de uma expiração máxima. As diferenças entre as duas medidas fornecem informações sobre o grau de expansibilidade torácica e abdominal.

A técnica de músculo energia foi realizada com a voluntária em posição sentada, com flexão de quadril e joelhos de $90^{\circ}$, pés totalmente apoiados no chão, coluna ereta, mãos entrelaçadas e posicionadas na região cervical. $\mathrm{O}$ terapeuta permanecia em posição bípede posteriomente à voluntária, com as mãos apoiadas nos da voluntária. Era então solicitado à voluntária que realizasse uma força contrária a resistência fornecida pela terapeuta durante 7 segundos, logo após a voluntária relaxava e a terapeuta então tracionava os braços posteriormente alongando a musculatura de peitoral. E esse procedimento foi realizado por 3 vezes.

A aplicação da técnica osteopática foi realizada pela mesma avaliadora, sendo realizada sempre no mesmo ambiente utilizando a mesma maca e o mesmo colchonete para toda a amostra avaliada, sendo que o indivíduo estava posicionado em decúbito dorsal, e o thrust foi aplicado na $6^{\mathrm{a}}$ vértebra torácica.

A manobra osteopática foi realizada posicionando a voluntária em decúbito dorsal com os membros inferiores estendidos, os membros superiores cruzados sobre o tronco anteriormente e cabeça rodada à esquerda. A terapeuta posicionada do lado direito da voluntária, e apoiando a região central e anterior de seu ombro, sobre o tórax da voluntária, com os dedos da mão direita fletidos, posicionados ao nível da sexta vértebra torácica. Terapeuta então eleva a paciente em flexão de tronco, abaixa-a, solicitando uma inspiração e no final da expiração terapeuta realiza um movimento brusco sobre os braços na parte central do tórax da voluntária (trush de $\mathrm{T}_{6}$ ).

Após a realização da técnica de músculo energia e da manobra de thrust, foi solicitado ao voluntário permanecer por 5 minutos em repouso, e então foi realizado novamente a avaliação dos parâmetros (cirtometria toracoabdo- 
minal e espirometria).

\section{Estatística}

Os resultados foram analisados inicialmente pela estatística descritiva (média e desvio padrão) e em seguida por meio do programa Statistica 6.0 (Stat Soft EUA), por meio do Teste de T-student para variáveis dependentes, com objetivo de identificar diferenças significativas $(p<0,05)$ entre as medidas coletadas antes da aplicação da técnica de manipulação de coluna tóracica e da técnica de músculo energia e no final do mesmo.

\section{Resultados e Discussão}

A caracterização da amostra estudada está disponível na tabela 1.

Tabela 1: Caracterização da amostra

\begin{tabular}{cccccc}
\hline & N & Média & Mínimo & Máximo & D.P \\
\hline IDADE & 30 & 20,800 & 18,000 & 28,000 & 1,901 \\
PESO & 30 & 55,533 & 47,000 & 69,000 & 5,952 \\
ALtURA & 30 & 1,653 & 1,510 & 1,750 & 0,059
\end{tabular}

Fonte: Dados do estudo

Legenda: N (amostra); D.P (devio padrão).

Os resultados obtidos por meio da espirometria e cirtometria toracoabdominal e suas comparações estão descritos na tabela 2 .

Tabela 2: Mensuração das variáveis

\begin{tabular}{lccc}
\hline & Média & D.P & p \\
\hline CVF_I \% & 73,900 & 13,939 & \\
CVF_F \% & 74,700 & 15,953 & 0,501 \\
VEF1_I \% & 72,567 & 16,228 & \\
VEF1_F\% & 74,667 & 17,085 & 0,128 \\
IT_I & 85,980 & 9,700 & \\
IT_F & 87,473 & 6,890 & 0,110 \\
PFE_I & 5,325 & 1,944 & \\
PFE_F & 5,726 & 1,951 & $0,047^{*}$ \\
VVM_I & 123,033 & 31,329 & \\
VVM_F & 126,133 & 33,706 & 0,151 \\
CA_I & 5,517 & 1,355 & \\
CA_F & 6,167 & 1,505 & $0,0002^{*}$ \\
CX_I & 6,283 & 2,340 & \\
CX_F & 6,783 & 2,024 & $0,0026^{*}$ \\
CAB_I & 3,600 & 1,289 & \\
CAB_F & 4,050 & 1,476 & $0,0051^{*}$ \\
\hline
\end{tabular}

Fonte: Dados do estudo

Legenda: CVF_I (capacidade vital forçada inicial); CVF_F (capacidade vital forçada final); $\mathrm{VEF}_{1-} \mathrm{I}$ (volume expiratório forçado no primeiro segundo inicial); $\mathrm{VEF}_{1} \mathrm{~F}$ (volume expiratório forçado no primeiro segundo final); IT_I (Indice Tiffenau inicial); IT_F (Índice Tiffenau final); PFE I (pico de fluxo expiratório inicial); PFE F (pico de fluxo expiratório final); VVM_I (ventilação voluntária máxima inicial); VVM_F (ventilação voluntária máxima final); CA_I (cirtometria axilar inicial); CA_F (cirtometria axilar final); CX_I (cirtometria xifoideana inicial); CX_F (cirtometria xifoideana final); CAB I (cirtometria abdominal inicial); CAB F (cirtometria abdominal final).

* Diferenças estatísticamente significante com $\mathrm{p}<0,05$.
O objetivo deste trabalho foi determinar se as manobras osteopáticas interferem na mobilidade e função pulmonar de indivíduos adultos saudáveis, para tanto foi utilizada manobras de thrust na sexta vértebra torácica e aplicada técnicas de músculo energia para peitorais, pois não está claro na literatura como tal interação pode influenciam a distribuição dos volumes, capacidades, e velocidade dos fluxos no interior dos pulmões, assim como também na mobilidade da caixa torácica.

Entretanto, são escassos os estudos que comprovam os benefícios da manipulação de vértebras torácicas e técnica de músculo-energia no que se refere ao incremento da função respiratória, contudo de maneira indireta vários estudos contribuem, relatando que técnicas de manipulação resultam em alongamento transitório de cápsulas das articulações, uma vez que envolve uma alta velocidade de impulso, onde as articulações são ajustadas rapidamente, permitindo que o sistema funcione otimamente e melhore a eficiência do organismo (ERNST, 2007).

Dessa forma, a manipulação espinal ou seu ajuste é um tratamento manual em que um conjunto vertebral é passivamente manipulado de maneira a promover o arco de movimento normal da vértebra, melhorando a mobilidade articular, como descreve Vernon e Mrozek (2005) em seus achados, corroborando com os resultados apresentados neste estudo, no qual constatou-se a melhora da mobilidade da caixa torácica após a manobra de thurst na coluna torácica.

Durante a mobilização/manipulação, os tecidos capsuloligamentar de uma articulação são alongados mecanicamente. Um objetivo principal da mobilização/manipulação é melhorar a extensibilidade do tecido capsuloligamentar restrito; secundariamente, o nível de ativação dos mecanorreceptores articulares é afetado. A mobilização articular tem demonstrado melhora nos movimentos fisiológicos e acessórios para a hipomobilidade de estruturas (MAKOFSKY et al., 2007).

Teoricamente com a melhora da mobilidade articular da caixa torácica que foi comprovada por meio deste estudo, outros efeitos na função pulmonar deveriam ser observados, porém neste estudo verificou-se apenas melhora ao exame de espirometria, do pico de fluxo expiratório (fase imediata), isso poderia ser explicado devido à amostra ser reduzida, já que os dados obtidos aumentavam progressivamente, entretanto não estatisticamente significantes.

Também foi constatada neste estudo que compilando os dados, a caracterização da amostra foi de elementos com caráter restritivo, todavia em um estudo realizado por Almeida et al. (2007), foi verificado que a sua amostra apresentou elementos com padrão obstrutivo, sendo que 41,7\% dos casos não foi possível justificar a obstrução brônquica, uma vez que esses indivíduos não eram fumantes e não tinham patologia prévia, nem exposição ocupacional ou de lazer, em nosso estudo a caracterização da nossa amostra foi de elementos com caráter restritivo, em contrapartida aos resultados obtidos em Portugal, isto explica-se pelo fato das síndromes restritivas terem sua origem no funcionamento inadequado dos centros nervosos e do aparelho muscular que comanda a ventilação, bem como na impossibilidade do parênquima pulmonar e da caixa torácica de se retrair e distender, mesmo quando adequadamente acionado pelo comando neuromuscular baseado em dados fornecidos por Gutiérrez 
(2006), podendo isto estar associado intimamente ao sedentarismo, visto que as voluntárias não tinham a atividade física como prática regular.

Para Suki et al. (2000), no pulmão normal, a capacidade residual funcional, representa um maior volume do pulmão do que o volume de fechamento, daí, durante a respiração normal, o fim do volume pulmonar expiratório não chega ao volume de fechamento, e o encerramento não ocorre, justificando a característica encontrada em nossa amostra neste estudo. Outra suposição é de que a fraqueza dos músculos produz um padrão restritivo na espirometria (SHAHRIZAILA; KINNEAR; WILLS, 2006).

Baseado em dados fornecidos por Grinnan e Truwit (2005), na fisiologia respiratória, a redução da complacência pulmonar é influenciada por uma parede torácica rígida ou capacidade de distensão e elastância pulmonar alterados, ou ambos. Assim para os mesmos autores a ventilação envolve o movimento da parede torácica para produzir um gradiente de pressão que irá permitir o fluxo e a troca gasosa.

Anogeianaki, Negrev e Ilonidis (2007) relatam que, acima de um determinado nível do esforço expiratório, o fluxo expiratório é exclusivamente devido à pressão de recolhimento elástico, em nossa amostragem observou-se aumento nesse indicador, o que é comprovado pela melhora da mobilidade da caixa torácica.

No entanto, em um modelo recente, em indivíduos normais mostrou que o pico de fluxo expiratório foi limitada pelas propriedades mecânicas do pulmão (em conformidade com o mecanismo de velocidade da onda), e não pela força muscular (TANTUCCI et al., 2002), o que enfatiza o nosso estudo.

O pico de fluxo expiratório é um parâmetro expiratório de esforço dependente, que reflete o calibre das vias aéreas proximais, a dependência do esforço torna a medida do PFE um índice interessante para analisar a colaboração na fase precoce da expiração, na qual avalia-se a pressão transpulmonar máxima, o pico de fluxo expiratório pode ainda ser utilizado como um índice indireto da capacidade da força expiratória (PEREIRA, 2002).

Em indivíduos saudáveis o pico de fluxo expiratório é determinado pelo volume dos pulmões (que é uma função das dimensões do tórax e, portanto, de estatura), pelas propriedades elásticas do pulmão, e pelo poder e coordenação dos músculos respiratórios, e ainda é dependente do esforço porque quanto maior o esforço durante a expiração precoce, isso faz, com que o fluxo eleve o nível do volume pulmonar, onde a complacência pulmonar é maior (QUANJER et al., 1997), o que ratifica os achados deste estudo, em que houve melhora na circunferência toracoabdominal, permitindo maior complacência pulmonar.

A anormal elasticidade do tecido pulmonar tem um impacto importante sobre a evolução clínica e resultado de muitas doenças pulmonares, no entanto, pouco se sabe sobre como as alterações nos componentes moleculares do tecido conjuntivo do pulmão afetam as suas propriedades mecânicas (CAVALCANTE et al., 2005). Sabe-se que a carga primária do tecido pulmonar apresenta três componentes que são elastina, colágeno e proteoglicanos, contudo, as interações entre esses componentes moleculares da tecido conjuntivo e como eles afetam a função pulmonar não está bem compreendido para os mesmos autores, acredita-se que em nossa amostra não há comprometimento da elasticidade do tecido pulmonar, uma vez que melhorado a expansibilidade torácica, houve resultado significativo no pico de fluxo expiratório.

O desequilíbrio na mecânica respiratória é geralmente decorrente de encurtamento excessivo da musculatura inspiratória e a força muscular apresenta-se alterada quando o músculo encontra-se em estado de encurtamento (TEODORI, 2003), o que gera alterações na capacidade pulmonar total.

Alongamento é tradicionalmente utilizado para aumentar a flexibilidade sobre um conjunto na tentativa de promover uma melhora na performance (MAREK et al., 2005), e é em torno da repartição das fibras conjuntivas e elásticas que gira o problema das retrações. Tal perda de elasticidade limita progressivamente o papel dos sarcômeros (COUTINHO; GOMES, 2004). Isso explica que, com o alongamento da musculatura de peitorais há uma melhora indiretamente da mobilidade da caixa torácica, promovendo consequentemente uma melhora da função pulmonar.

Em nossos achados a melhora da velocidade com que o ar é expelido dos pulmões pode ser devido à diminuição da tensão sobre as costelas decorrente dos músculos intercostais, da parede abdominal e das fáscias musculares, e consequentemente melhora da força muscular, nesse sentido o aumento de força muscular em função do alongamento deve-se possivelmente a melhor interação entre os filamentos de actina e miosina, em virtude do aumento do comprimento funcional do músculo (WELDON; HILL, 2003).

Kakizaki et al. (1999) mostraram que o alongamento dos músculos respiratórios em portadores de DPOC levou a aumento da CVF. A CVF é dependente do recolhimento elástico dos pulmões, da elastância da caixa torácica e da força dos músculos respiratórios, assim exercícios de alongamento da musculatura respiratória não devem alterar a estrutura do tecido pulmonar. Assim, é possível que os exercícios realizados tenham modificado a elastância da caixa torácica ou a força dos músculos respiratórios. Neste estudo, foi observado aumento da mobilidade torácica sem alteração da CVF. Embora ainda não seja possível uma explicação mais aprofundada sobre os resultados obtidos na análise da mobilidade torácica e da função pulmonar, espera-se que estudos futuros possam esclarecer melhor essa aparente discrepância.

Outra hipótese plausível de investigação, é a da existência de um ritmo circadiano na função pulmonar, já bem reconhecido, sendo exposto por Medarov, Pavlov e Rossoff (2008), que demonstraram em 4756 espirometrias de indivíduos saudáveis, dados de VEF1, CVF e VEF1/CVF e PFE estatisticamente significativos de variações diurnas para as quatro variáveis, a menor média observadas no intervalo das 12:00-12:59 da tarde, os maiores valores médios foram identificados na parte da tarde das 15:00-15:59 e 16:00-16:59, dois outros picos menores (das 8:00-8:59 e 11:00-11:59 da manhã), em que este é um ponto a ser investigado em futuras pesquisas, já que estes intervalos não foram considerados durante a avaliação das espirometria desta amostra, embora o ritmo circadiano seja reconhecido pelos pesquisadores.

Sugere-se novas pesquisas sobre o assunto, com abordagens mais complexas, indicando o uso de outras técnicas voltadas para ativação de diafragma, alongamento de musculatura acessória, e cuja amostragem seja maior, uma vez que uma amostra reduzida ocasiona a heterogeneidade 
dos dados.

\section{Conclusão}

O protocolo desenvolvido durante este estudo foi capaz de aumentar a mobilidade torácica em todos os parâmetros avaliados, assim como também foi relevante na melhora do pico de fluxo expiratório observado durante a espirometria, entretanto não foi capaz de alterar os demais parâmetros avaliados neste teste.

Com o presente estudo pode-se observar que as técnicas osteopáticas foram eficientes na melhora da mobilidade e na função pulmonar, e só não foram evidentes em mais parâmetros avaliados devido a amostra ser reduzida, entretanto esta pesquisa elucida que as técnicas quando aplicadas em coluna vertebral intervêm na função pulmonar.

\section{Agradecimentos}

Os autores agradecem o apoio do Programa de Iniciação Científica da Universidade Paranaense UNIPAR, o qual foi o financiador do mesmo.

\section{Referências}

ALMEIDA, A. G. Função respiratória em bombeiros portugueses. Rev. Port. de Pneumol., v. 13, n 3, p. 349364, 2007.

ANOGEIANAKI, A.; NEGREV, N., ILONIDIS, G. Contributions of signal analysis to the interpretation of spirometry. HIPPOKRATIA, v. 11, n. 4, p. 187-195, 2007.

BIENFAIT, M. Os desequilíbrios estáticos. 3 ed. São Paulo: Summus, 1995.

CALDEIRA, V. da S. et al. Precisão e acurácia da cirtometria em adultos saudáveis. Jornal Bras. Pneumol., v. 33, n. 5, 2007.

CAVALCANTE, F. S. A. et al. Mechanical interactions between collagen and proteoglycans: implications for the stability of lung tissue. J Appl Physiol, v. 98, p. 672-679, 2005.

CLANTON, T.L. LEVINE S. Respiratory muscle fiber remodeling in chronic hyperinflation: dysfunction or adaptation? J Appl Physiol. v. 107, p. 324-35. 2009.

CLELAND, J. A.; et al. The Audible Pop from Thoracic Spine Thrust Manipulation and Its Relation to Short-Term Outcomes in Patients with Neck Pain. The Journal of Manual \& Manipulative Therapy, v. 15, n. 3, p. 143-154, 2007.

COSTA, D. et al. Estudo dos volumes pulmonares e da mobilidade toracoabdominal de portadoras de obesidade mórbida, submetidas à cirurgia bariátrica, tratadas com duas diferentes técnicas de fisioterapia. Rev. Bras. Fisioter., v.13, n.4, 2009.
COUTINHO, E. L.; GOMES, A. R. S. Effect of passive stretching on the immobilized soleus muscle fiber morfology. Brazilian Journal of Medical and Biological Research, n. 37, p. 1853-1861, 2004.

ERNST, E. Adverse effects of spinal manipulation: a systematic review. J R Soc Med, v. 100, p. 330-338, 2007.

FARKAS, G. A.; CERNY, F. J.; ROCHESTER, D.F. Contractility of the ventilatory pump muscles. Med Sci Sports Exerc, v. 28, n 9, p. 1106-14, 1996.

GRINNAN, D. C.; TRUWIT, J. D. Clinical review: Respiratory mechanics in spontaneous and assisted ventilation. Critical Care, v. 9, n. 5, p. 472- 484, 2005.

GUTIÉRREZ, M. C. Evaluación de diferentes valores de referencia espirométricos para el diagnóstico de alteración restrictiva en población chilena. Rev Chil Enf Respir, v. 22, p. 86-92. 2006.

\section{GUYTON, A. C.; HALL, J. E. Tratado de fisiologia} médica. 10 ed. Rio de Janeiro: Guanabara Koogan, 2002.

HENLEY, C. E. et al. Osteopathic manipulative treatment and its relationship to autonomic nervous system activity as demonstrated by heart rate variability: a repeated measures study. Osteopathic Medicine and Primary Care, v. 2, n. 7 , p. 1-8, 2008.

KAKIZAKI, F.; et al. Preliminary report on the effects of respiratory muscle stretch gymnastics on chest wall mobility in patients with chronic obstructive pulmonary disease. Respir Care, v. 44, p. 409-14, 1999.

KAPANDJI, A. I. Fisiologia Articular. 5 ed. Rio de Janeiro: Panamericana, v.3, 2000.

KENDALL, F. P.; McCREARY, E. K.; PROVANCE, P. G. Músculos: Provas e funções. São Paulo: Manole, 1995.

LEITE, P. F. Fisiologia do exercício: ergometria e condicionamento físico. São Paulo: Probael, 2000.

LOMBARDINI $\mathrm{R}$ et al. The use of osteopathic manipulative treatment as adjuvant therapy in patients with peripheral arterial disease. Manual Therapy. v.14, n.4, p 439-443, 2009 .

MAKOFSKY, H. et al. Immediate Effect of Grade IV Inferior Hip Joint Mobilization on Hip Abductor Torque: A Pilot Study. The Journal of Manual \& Manipulative Therapy, v. 15, n 2, p. 103-111, 2007.

MAREK, S. M. et al. Acute Effects of Static and ProprioceptiveNeuromuscular Facilitation Stretching on Muscle Strength and Power Output. Journal of Athletic Training, v. 40, n 2, p. 94-103, 2005.

MEDAROV, B. I.; PAVLOV, V. A.; ROSSOFF, L. Diurnal Variations in Human Pulmonary Function. Int J Clin Exp 
Med, v. 1, p. 267-273, 2008.

OCHS, M. et al. The number of alveoli in the human lung. Am J Respir Crit Care Med, v. 169, p. 120-4, 2004.

PEREIRA, C. A. de C. Espirometria. Jornal de

Pneumologia, v.28, supl. 3, p. 1-81, 2002.

QUANJER, P. H. et al. Peak expiratory flow: conclusions and recommendations of a Working Party of the European Respiratory Society. Eur Respir J, v. 10, supl. 24, p. 2s-8s, 1997.

SHAHRIZAILA, N.; KINNEAR, W. J. M.; WILLS, A. $\mathrm{J}$. Respiratory involvement in inherited primary muscle conditions. J Neurol Neurosurg Psychiatry, v. 77, p. 1108-1115, 2006.

SILVA, L. C. C. da. Espirometria na prática médica. Revista AMRIGS, v.49, nº 3, p.186-94, 2005.

SOUCHARD, E. O diafragma: anatomia, biomecânica, bioenergética, patologia, abordagem terapêutica. 2 ed. São Paulo: Summus, 1980.

SUKI, B. et al. Size distribution of recruited alveolar volumes in airway reopening. J Appl Physiol, v. 89, p. 2030-2040, 2000.

TANTUCCI, C. et al. The best peak expiratory flow is flowlimited and effort-independent in normal subjects. Am J Resp Crit Care Med, v. 165, p. 1304-1308, 2002.

TEODORI, R. M. et al. Alongamento da musculatura inspiratória por intermédio da reeducação postural global (RPG). Rev. Bras. Fisioter., v. 7, n. 1, p. 25-30, 2003.

THOMAS, J. R.; NELSON, J. K. Métodos de pesquisa em atividade física. 3.ed. Porto Alegre: Artmed, 2002.

VERNON, H.; MROZEK, J. A revised definition of manipulation. J Manip Physiol Ther, v. 28, p. 68-72, 2005.

YAMAGUTI, Wellington Pereira dos Santos et al. Avaliação ultra-sonográfica da mobilidade do diafragma em diferentes posturas em sujeitos saudáveis. J. bras. pneumol. 2007, vol.33, n.4, pp. 407-413

WELDON, S. M.; HILL, R.H. The efficacy of stretching for prevention of exercise related injury: a systematic review of thev literature. Manual Therapy, v. 8, n. 3, p. 141-150, 2003. 